\title{
Balloon dilatation of pacemaker induced stenosis of the superior vena cava
}

\author{
A A Grace, M Sutters, P M Schofield
}

\begin{abstract}
A 53 year old woman with symptomatic pacemaker associated superior vena cava syndrome was treated successfully with balloon angioplasty. She was well six months after the procedure.
\end{abstract}

The superior vena cava syndrome is a well recognised but unusual complication of permanent pacemaker implantation. The syndrome results from the development of a stenosis in the superior vena cava with symptoms arising secondary to impaired regional venous drainage. Management is controversial and thoracotomy and caval reconstruction have been used to relieve symptoms. ${ }^{12}$ We report the successful use of balloon angioplasty as the primary treatment of this condition.

\section{Case report}

A 53 year old woman presented with a long history of severe palpitation. Atrioventricular nodal reentrant tachycardia was confirmed at electrophysiological study. The arrhythmia was refractory to medical treatment and in 1985 a PASAR (Telectronics) antitachycardia pacemaker was implanted. A Telectronics atrial lead was advanced to the right atrial appendage without difficulty. This relieved her symptoms considerably. A year after the procedure she presented with pleuritic chest pain and treatment with warfarin was started after a clinical diagnosis of pulmonary embolism. In 1988 she developed swelling of her hands and face and an angiogram showed stenosis of the superior vena cava proximal to its entry into the right atrium. Pulmonary artery pressure was normal and a pulmonary angiogram showed no evidence of pulmonary embolism. No action was taken. By 1990 the swelling of her upper body, which was especially severe in the morning and was made worse by activity, had increased and in addition she was experiencing worsening headaches. There was mild pitting oedema of the arms. The jugular vein was non-pulsatile and visible beyond the angle of the jaw. The electrocardiogram showed minor repolarisation abnormalities and the chest $x$ ray was normal. Computed tomography showed a normal mediastinum. A repeat angiogram of the superior vena cava showed that the stenosis was more severe than before (fig 1). There was a gradient of $9 \mathrm{~mm} \mathrm{Hg}$ between the superior vena cava above the stenosis and the right atrium. The angiographic appearance was suggestive of a fibrotic stricture with no evidence of associated thrombus. Contrast injections into the cephalic vein in the left antecubital fossa showed another stenosis at the junction of the brachiocephalic and the subclavian veins on that side. Multiple collaterals were also present. Balloon angioplasty of the stenosis of the superior vena cava was undertaken. A 0.035 inch straight guide wire and $7 \mathrm{~F}$ Gensini catheter were introduced from the right femoral vein and the wire was passed through the stenosis to the right subclavian vein. The catheter was then advanced through the stenosis to allow a 0.035 inch exchange $J$ guide to be introduced. The stenosis was then dilated with an $8 \mathrm{~mm}$ balloon catheter initially and then with a $10 \mathrm{~mm}$ balloon catheter (Mansfield). A good angiographic result was obtained (fig 2) and the gradient across the stenosis was abolished. The procedure was uneventful apart from two brief episodes of supraventricular tachycardia, during which the pacing unit continued to function normally. On examination the next day the jugular vein was no longer visible above the sternal angle. She remained symptom free six months after the procedure.

\section{Discussion}

Though venous abnormalities induced by pacemaker leads are common, ${ }^{3}$ stenosis of the superior vena cava and associated symptoms are unusual. The condition is more common

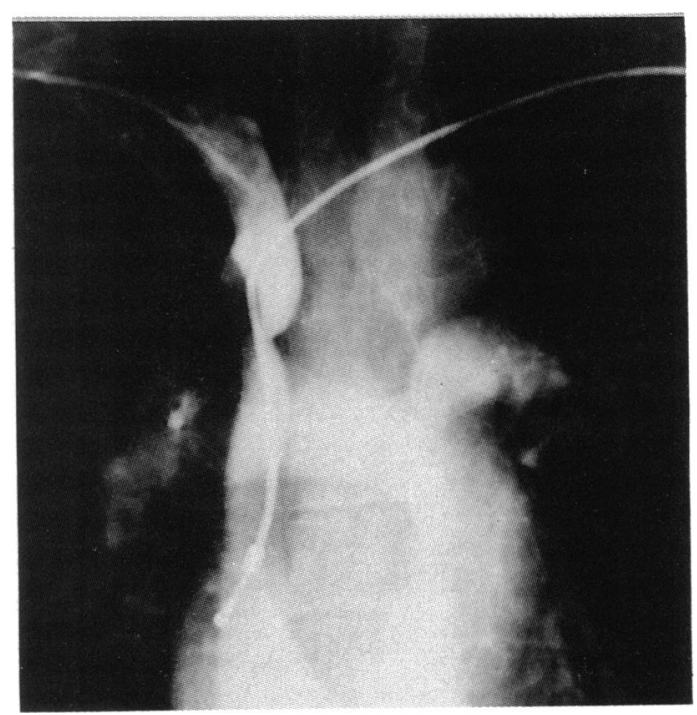

Figure 1 Dye injection into the right subclavian vein showing stenosis of the the superior vena cava above its junction with the right atrium. The Teletronics atrial lead can be seen in position.
Papworth Hos Cambridge

A A Grace 
Figure 2 Angiogram after balloon dilatation showing considerable improvement in flow.

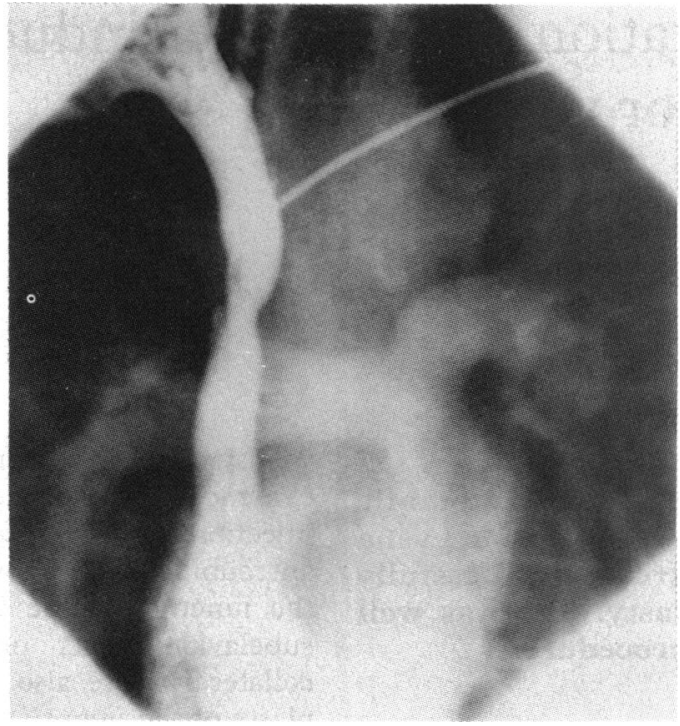

in patients with retained leads and in those with infectious complications after pacemaker implantation ${ }^{1}$ and is the result of thrombosis, which may be associated with fibrosis. Ideal management is not established but thrombotic occlusion may be reduced by the administration of intravenous thrombolytic agents. Several patients have been given oral anticoagulants but the risk of resultant cerebral haemorrhage is probably increased by coexistent cerebral venous hypertension. ${ }^{45}$ Thoracotomy and caval repair have also been undertaken when symptoms persisted and a stenosis was shown on angiography. There are reports of the use of percutaneous balloon angioplasty in two previous patients. In one it was used in conjunction with thrombolytic treatment ${ }^{6}$ and in the other for restenosis after caval repair in which a pericardial patch was inserted."

Our patient is the first in whom balloon dilatation was used successfully as a primary treatment for stenosis of the superior vena cava: a stenosis of an innominate vein has been dilated and reported previously. ${ }^{7}$ The stenosis that we dilated seemed to be fibrotic, and not associated with thrombus; it may have resulted from damage at the time of introduction of the pacing lead. The angioplasty technique we used was standard and no appreciable problems were encountered. We expect that any complications are likely to be those associated with vascular access, lead displacement, and caval rupture. Restenosis has been reported ${ }^{1}$ but the likely incidence cannot be determined from the available reports.

Because the risk of cerebral haemorrhage is likely to be increased by the use of anticoagulants we recommend balloon angioplasty as the primary treatment for fibrotic strictures occurring in this unusual condition. Thrombolytic agents should be used only when coexistent thrombus is visible on angiography and they should be used with caution.

A A G is supported by the British Heart Foundation.

1 Goudevenos JA, Reid PG, Adams PC, Holden MP, Williams DO. Pacemaker induced superior vena cava syndrome: report of four cases and review of the literature. $P A C E$ 1989;12:1890-5

2 Blackburn T, Dunn M. Pacemaker induced superior vena cava syndrome: consideration of management. Am Heart $\mathrm{J}$ 1988;116:893-6.

3 Stoney WS, Addlestone RB, Alford WC, et al. The incidence of venous thrombosis following long term transvenous pacing. Ann Thorac Surg 1976;22:166-70.

4 Matthews DM, Forfar JC. Superior vena caval stenosis: a complication of transvenous endocardial pacing. Thorax 1979;34:412-3.

5 Pauletti M, Pingitore $R$, Contini C. Superior vena cava stenosis at site of intersection of two pacing electrodes. Br Heart J 1979;42:487-9.

6 Montgomery JH, D'Souza VJ, Dyer RB, et al. Nonsurgical treatment of the superior vena cava syndrome. Am J treatment of the superior
Cardiol $1985 ; 56: 829-30$.

7 Spittel PC, Vliestra RE, Hayes DL, Higano ST. Venous obstruction due to permanent transvenous pacemaker electrodes: treatment with percutaneous transluminal balloon venoplasty. $P A C E$ 1990;13:271-4 